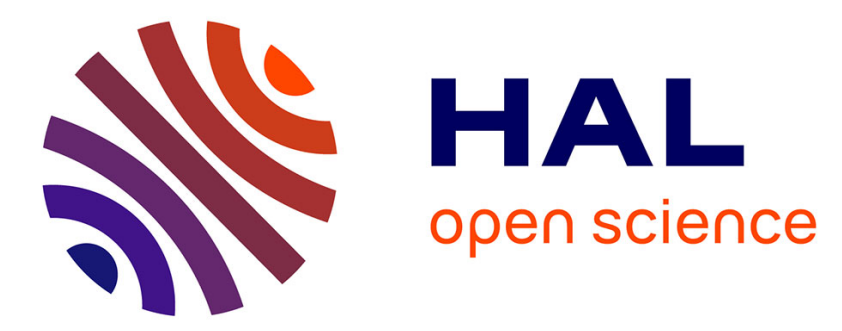

\title{
Probing Low-Energy Resonances in Water-Hydrogen Inelastic Collisions
}

\author{
A. Bergeat, Sébastien B. Morales, Christian Naulin, Laurent Wiesenfeld, A \\ Faure
}

\section{- To cite this version:}

A. Bergeat, Sébastien B. Morales, Christian Naulin, Laurent Wiesenfeld, A Faure. Probing LowEnergy Resonances in Water-Hydrogen Inelastic Collisions. Physical Review Letters, 2020, 125, 10.1103/physrevlett.125.143402 . hal-03058396

\section{HAL Id: hal-03058396 https://hal.science/hal-03058396}

Submitted on 11 Dec 2020

HAL is a multi-disciplinary open access archive for the deposit and dissemination of scientific research documents, whether they are published or not. The documents may come from teaching and research institutions in France or abroad, or from public or private research centers.
L'archive ouverte pluridisciplinaire HAL, est destinée au dépôt et à la diffusion de documents scientifiques de niveau recherche, publiés ou non, émanant des établissements d'enseignement et de recherche français ou étrangers, des laboratoires publics ou privés. 


\title{
Probing Low-Energy Resonances in Water-Hydrogen Inelastic Collisions
}

\author{
A. Bergeat $\odot,{ }^{*}$ S. B. Morales, and C. Naulin \\ Université de Bordeaux, CNRS, Bordeaux INP, ISM, UMR5255, F-33405 Talence, France \\ L. Wiesenfeld 1 \\ Université Paris-Saclay, CNRS, Laboratoire Aimé Cotton, F-91405 Orsay, France \\ A. Faure ${ }^{\circ}$ \\ Université Grenoble Alpes, CNRS, IPAG, F-38000 Grenoble, France
}

(Received 5 June 2020; accepted 17 August 2020; published 30 September 2020)

\begin{abstract}
Molecular scattering at collisional energies of the order of $10-100 \mathrm{~cm}^{-1}$ (corresponding to kinetic temperatures in the $15-150 \mathrm{~K}$ range) provides insight into the details of the scattering process and, in particular, of the various resonances that appear in inelastic cross sections. In this Letter, we present a detailed experimental and theoretical study of the rotationally inelastic scattering of ground-state ortho$\mathrm{D}_{2} \mathrm{O}$ by ground-state para- $\mathrm{H}_{2}$ in the threshold region of the $\mathrm{D}_{2} \mathrm{O}\left(\mathrm{O}_{00} \rightarrow 2_{02}\right)$ transition at $35.9 \mathrm{~cm}^{-1}$. The measurements were performed with a molecular crossed beam apparatus with variable collision angle, thence with variable collisional energy. Calculations were carried out with the coupled-channel method combined with a dedicated high-level $\mathrm{D}_{2} \mathrm{O}-\mathrm{H}_{2}$ intermolecular potential. Our theoretical cross section $0_{00} \rightarrow 2_{02}$ is found to display several resonance peaks in perfect agreement with the experimental work, in their absolute positions and relative intensities. We show that those peaks are mostly due to shape resonances, characterized here for the first time for a polyatomic molecule colliding with a diatom.
\end{abstract}

DOI: 10.1103/PhysRevLett.125.143402

Introduction.-Water is the most abundant molecule in the Universe after $\mathrm{H}_{2}$ and $\mathrm{CO}$. As a polar polyatomic species, it acts as an efficient coolant for collapsing interstellar clouds but it also plays a key role as a reservoir species for oxygen and as a catalyst on the surface of interstellar dust grains. Water is observed ubiquitously in the Solar System, in the Milky Way, and in the far Universe. Because of its large abundance in our own atmosphere, the main isotopologue, ${ }^{1} \mathrm{H}_{2}{ }^{16} \mathrm{O}$, is difficult to detect from the ground, except for some strong maser lines [1]. As a result, the rich rotational and rovibrational spectrum of water has been essentially observed from space, thanks to the (far) infrared space missions ISO, SWAS, Odin, Spitzer, and Herschel [2]. Rare isotopologues of water have been also detected in interstellar clouds, including the doubly deuterated ${ }^{2} \mathrm{H}_{2}{ }^{16} \mathrm{O}$ [3], denoted $\mathrm{D}_{2} \mathrm{O}$ or heavy water hereafter.

In order to quantitatively model the physical and chemical conditions of astrophysical sources where water is detected, it is necessary to understand the mechanisms of spectral line formation, including position, shape, and intensity. The frequencies and radiative Einstein coefficients of many water transitions are known with high accuracy. However, because of the low interstellar densities and the competition between photonic and collisional processes, any radiative transfer model must also include a proper treatment of collisional excitation, while collisional line broadening and shifting are fully negligible.
It has long been recognized that only theory is capable of yielding precise enough values of the collisional rate coefficients $k_{i \rightarrow f}(T), i$ and $f$ being rotational or rovibrational states of water, because of the wide kinetic temperature range and the large number of $i, f$ states of relevance in astrophysical media. Huge progress was made over the last years thanks to dedicated and concerted efforts of theoreticians to provide rate coefficients for the rotational excitation of $\mathrm{H}_{2} \mathrm{O}[4,5], \mathrm{HDO}$, and $\mathrm{D}_{2} \mathrm{O}[6]$ by $\mathrm{H}_{2}$, which is the dominant collider in molecular clouds. All these calculations employed the full nine-dimensional $\mathrm{H}_{2} \mathrm{O}-\mathrm{H}_{2}$ potential energy surface (PES) computed by Valiron et al. [7], denoted V08 hereafter.

The main goal of the present work is to observe the lowenergy resonances in the water-molecular hydrogen system by measuring the rotationally inelastic scattering of ortho$\mathrm{D}_{2} \mathrm{O}$ by para- $\mathrm{H}_{2}$, and to compare those measured resonance peaks with a theoretical computation, performed at the best possible level of quantum dynamics. Resonances are predicted to be easier to detect with para- $\mathrm{H}_{2}$ than with ortho- $\mathrm{H}_{2}$, thanks to the smaller number of overlapping resonant features. The use of $\mathrm{D}_{2} \mathrm{O}$ rather than $\mathrm{H}_{2} \mathrm{O}$ also greatly improves the signal-to-noise ratio. Because the positions and amplitudes of the scattering resonances are extremely sensitive to the finer details of the underlying PES, in particular its long-range anisotropy, this measurement provides the ultimate test of the V08 PES. Thus, good 
agreement with a fully converged theoretical computation would give considerable confidence in the observables derived from this PES, such as the collisional rate coefficients or the spectrum of hydrogen molecules in clathrate hydrates [8].

Many previous experimental tests of the V08 potential exist, which have probed its short-range and well regions. These include elastic integral cross sections [9], second virial coefficients [10], bound states of the complex (Ref. [11] and references therein) and pressure broadening rates [12]. A number of differential scattering measurements are also available at the state-to-state level. The first of such studies [13] appeared in 2010 where relative stateto-state differential cross sections (DCSs) for $\mathrm{H}_{2} \mathrm{O}-\mathrm{H}_{2}$ were measured at $575 \mathrm{~cm}^{-1}$ collisional energy, and later at $361 \mathrm{~cm}^{-1}$ [14]. A similar experimental study was also performed for the water isotopologues $\mathrm{HDO}$ and $\mathrm{D}_{2} \mathrm{O}$ [15]. The agreement with theoretical DCSs was generally good but these relatively high collisional energies and the coexistence of several rotational states of $\mathrm{H}_{2}\left(j_{2}=0,1,2\right)$ made the comparison not fully conclusive. The comparison of absolute values of theoretical state-to-state collisional rate coefficients with experimental data is available for the water-hydrogen-molecule system only via the detour of pressure broadening rates [12].

More recently, Bergeat et al. [16] have measured relative integral cross sections (ICSs) for $\mathrm{H}_{2} \mathrm{O}$ colliding with normal- $\mathrm{H}_{2}$ at much lower collisional energies, in the range $15-105 \mathrm{~cm}^{-1}$. The agreement with theory was found to be very good. The lack of any observable structure on the excitation function could not provide, however, a full experimental check of the long-range anisotropy of the V08 PES, because of the use of normal- $\mathrm{H}_{2}\left(j_{2}=0,1\right)$ and the background signal.

Experiment.-We performed experiments in a crossed molecular beam apparatus [16,17], with variable collisional angle, under single collision conditions. A pulse of $\mathrm{D}_{2} \mathrm{O}$ seeded in helium was generated with an Even-Lavie valve maintained at $320 \mathrm{~K}$. As the best collisional energy resolution is obtained when the two beams have almost the same velocity, for the para $\mathrm{H}_{2}$ beam, a cryogenically cooled valve was used (see the Supplemental Material for details). The para- $\mathrm{H}_{2}$ was produced by liquefaction of normal- $\mathrm{H}_{2}$ on a $\mathrm{NiSO}_{4}$ catalyst using a He-closed cycle cryostat. The reactants and the scattered heavy water were state-selectively detected in the crossing region using a time-of-flight mass spectrometer after resonance-enhanced multiphoton ionization (REMPI). Spectra of normal- and para- $\mathrm{H}_{2}$ beams (see Fig. 3 in the Supplemental Material) show that the para- $\mathrm{H}_{2}$ beam composition remains almost the same during the experiment: the $j_{2}=1$ line remains within the background noise, which should correspond to a $j_{2}$ rotational population ratio $(1 / 0)$ below 0.15 . The rotational distribution of $\mathrm{D}_{2} \mathrm{O}$ was evaluated (Fig. 1) with a simulation by PGOPHER [18] and the spectroscopic data of Ref. [19]. The ortho/para ratio of

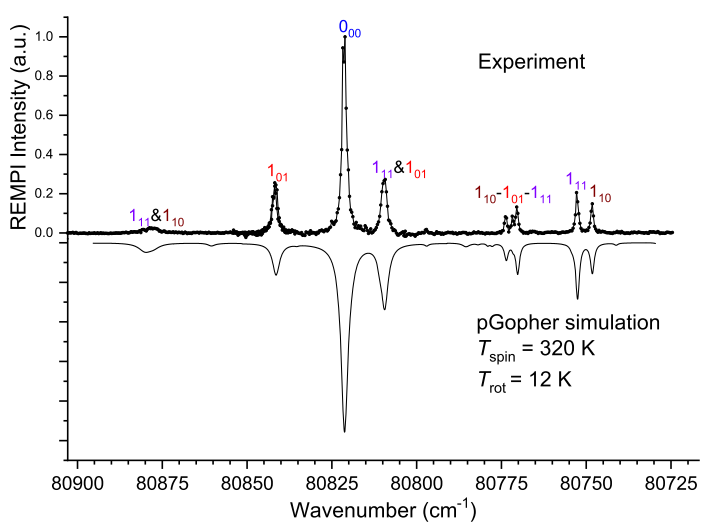

FIG. 1. $(2+1)$ REMPI spectrum of the $\tilde{\mathrm{C}}^{1} \mathrm{~B}_{1}, v^{\prime}=0 \leftarrow \tilde{\mathrm{X}}^{1} \mathrm{~A}_{1}$, $v=0$ transition of $\mathrm{D}_{2} \mathrm{O}$ in the supersonic beam (top) and a simulated spectrum for a beam of normal $\mathrm{D}_{2} \mathrm{O}$ with a rotational temperature of $12 \mathrm{~K}$ (bottom). The labels indicate the rotational levels, $j_{k_{a} k_{c}}$ from which the transitions originate. The ortho- $\mathrm{D}_{2} \mathrm{O}$ probed states are specified in blue for the ground state and in violet for the first excited state. The para- $\mathrm{D}_{2} \mathrm{O}$ probed states are in red for the ground state and in purple for the first excited state. See Ref. [19] for more details.

$\mathrm{D}_{2} \mathrm{O}$ remains at the room temperature equilibrium ratio of 2 . For ortho and para states separately, the REMPI signals show that $\mathrm{D}_{2} \mathrm{O}$ is mainly in its ground rotational levels and with a small amount in the first excited rotational levels: we estimated the $j_{k_{a} k_{c}}$ rotational population ratios $\left(0_{00} / 1_{11}\right) \sim$ $0.81 / 0.19$ and $\left(1_{01} / 1_{10}\right) \sim 0.73 / 0.27$.

The experimental inelastic cross section for the rotational excitation of $\mathrm{D}_{2} \mathrm{O}, \sigma\left(E_{\text {coll }} ; 0_{00} \rightarrow 2_{02}\right)$, by para- $\mathrm{H}_{2}$ is shown in Fig. 2 as a function of the collisional energy $E_{\text {coll }}$, i.e., the relative translational energy of the collision partners.

Theory.-The inelastic scattering calculations were performed with the MOLSCAT program [20] by combining the $\mathrm{V} 08 \mathrm{H}_{2} \mathrm{O}-\mathrm{H}_{2}$ PES with the coupled-channel method. The full nine-dimensional V08 PES is independent of nuclear masses and can be employed for any water and molecular hydrogen isotopologue. Full details about the transformation from $\mathrm{H}_{2} \mathrm{O}-\mathrm{H}_{2}$ to $\mathrm{D}_{2} \mathrm{O}-\mathrm{H}_{2}$ can be found in the work of Scribano et al. [21,22]. The intermolecular coordinates are defined in the center-of-mass frame of $\mathrm{D}_{2} \mathrm{O}$ and the internal coordinates of $\mathrm{D}_{2} \mathrm{O}$ and $\mathrm{H}_{2}$ are fixed at their vibrationally averaged values. We note that the rigid-rotor approximation was recently validated thanks to scattering calculations that include the $\mathrm{H}_{2} \mathrm{O}$ bending-vibrational motion [23].

The rotational energy levels of $\mathrm{D}_{2} \mathrm{O}$ are labeled either by three numbers, the angular momentum $j_{1}$ and the pseudoquantum numbers $k_{a}$ and $k_{c}$ (corresponding to the projection of $j_{1}$ along the principal inertia $a-$ and $c$-axes) or alternatively, by the quantum numbers $j_{1}$ and $\tau_{1}=k_{a}-k_{c}$. Ortho- $\mathrm{D}_{2} \mathrm{O}$ levels are characterized with $k_{a}+k_{c}$ even. The rotational levels of para- $\mathrm{H}_{2}$ are labeled by the angular momentum $j_{2}=0,2, \ldots$ The rotational constants were taken as $A=15.41998, B=7.27299$, and $C=4.84529 \mathrm{~cm}^{-1}$ for $\mathrm{D}_{2} \mathrm{O}$ and $B_{0}=59.322 \mathrm{~cm}^{-1}$ for $\mathrm{H}_{2}$, as in Ref. [21]. 


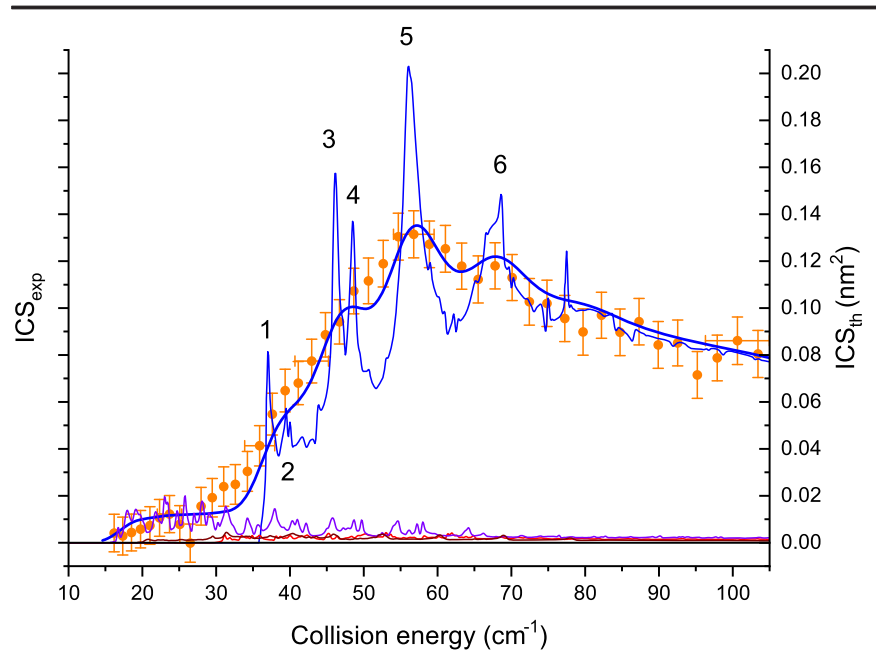

FIG. 2. Comparison of experimental and theoretical integral cross sections for the rotational excitation of ortho- $\mathrm{D}_{2} \mathrm{O}\left(\mathrm{O}_{00} \rightarrow\right.$ $\left.2_{02}\right)$ by para- $\mathrm{H}_{2}\left(j_{2}=0\right)$. Orange points, experimental cross sections (arbitrary units, left $y$ axis). Vertical error bars represent statistical uncertainties at $95 \%$ of the confidence interval. Horizontal error bars represent uncertainties in energy calibration. Thin blue line, theoretical fully converged ICS (in $\mathrm{nm}^{2}$, right $y$ axis) for the $0_{00} \rightarrow 2_{02}$ excitation. Main peaks are labeled 1-6. Thick blue line, the overall theoretical data convoluted with the experimental energy resolution; smaller theoretical contributions, scaled to their experimental weights relatively to the $\mathrm{D}_{2} \mathrm{O}\left(\mathrm{O}_{00}\right)$ population-see text: violet, $1_{11} \rightarrow 2_{02}$ (weight of 0.23 ), red $1_{01} \rightarrow 2_{12}(0.09)$, and purple, $1_{10} \rightarrow 2_{12}(0.03)$.

The reduced mass of $\mathrm{D}_{2} \mathrm{O}-\mathrm{H}_{2}$ is $1.8313 u$. The coupled differential scattering equations were solved using the hybrid modified log-derivative Airy propagator. Total energies up to $200 \mathrm{~cm}^{-1}$ were considered using a fine energy grid of $0.25 \mathrm{~cm}^{-1}$ for a proper description of resonances. Calculations were performed with $\mathrm{H}_{2}$ in its ground para state $\left(j_{2}=0\right)$. The highest rotational angular momentum of ortho$\mathrm{D}_{2} \mathrm{O}$ in the basis set was $j_{1}=6$ (corresponding to the inclusion of 25 levels), whereas the lowest two rotational levels of para- $\mathrm{H}_{2}$ were included in the basis $\left(j_{2}=0,2\right)$. Extending rotational bases beyond those limits was shown to be insignificant. The inclusion of many closed channels is essential to properly describe the transient excitation of $\mathrm{D}_{2} \mathrm{O}$ because of the deep well of the $\mathrm{D}_{2} \mathrm{O}-\mathrm{H}_{2}$ PES $\left(D_{e}=238 \mathrm{~cm}^{-1}\right)$ and its very anisotropic nature. Convergence was also checked as a function of the propagation parameters. Finally, both integral and differential cross sections were computed as a function of the total angular momentum (partial wave) $J$, with $J$ ranging from 0 to 33, yielding full convergence.

Results.-As expected, the theoretical excitation function shown as a thin blue line in Fig. 2 exhibits a threshold behavior corresponding to the rotational energy difference of $35.9 \mathrm{~cm}^{-1}$ between the two rotational levels, $\mathrm{D}_{2} \mathrm{O}\left(2_{02}\right)$ and $\left(0_{00}\right)$. However, contrary to the theoretical calculations, the experimental excitation function (points) presents a signal below this threshold. One contribution is due to the presence of some $\mathrm{D}_{2} \mathrm{O}\left(1_{11}\right)$ (about 13\%) in the beam, as shown in Fig. 1. Moreover, at the wave number of $80860 \mathrm{~cm}^{-1}$ chosen to probe the $2_{02}$ rotational state, there is a small contribution from the $2_{12}$ level. Thus, the ICSs from the $1_{01} \rightarrow 2_{12}$ and $1_{10} \rightarrow 2_{12}$ were considered with weights taking into account the ortho/para ratio of $\mathrm{D}_{2} \mathrm{O}$ in the beam, the relative population of the $1_{10}$ and $1_{01}$ states and the relative strength of the REMPI transitions from the 202 and $2_{12}$ levels [19].

The four sets of the theoretical excitation functions are plotted in Fig. 1 of the Supplemental Material. They are displayed in Fig. 2, weighted to their relative contributions to the $2_{02}$ signal, under the experimental conditions. The overall theoretical excitation function is the sum of these four contributions. However, theory provides absolute values of $\sigma\left(E_{\text {coll }}\right)$, while experimental results are obtained in a relative scale. To allow for comparison with the experimental data, the overall theoretical results were convoluted with the experimental collisional energy spread (see Fig. 4 of Supplemental Material). The experimental data were then rescaled to have the same area as the theoretical ones in their common energy domain $\left(E_{\text {coll }}=30-100 \mathrm{~cm}^{-1}\right)$.

The agreement between theory and experiment is remarkable, both in energy and intensity, all the more so because the only free parameter is the overall scale of the experimental cross section. Not only is the overall shape of the inelastic cross section fully reproduced, but also the broadened resonances are faithfully reproduced (in amplitude and position) thanks to the convolution of the sharp theoretical resonances, whose intensities are typically twice that of the background cross section. The six main peaks are numbered in Fig. 2. They correspond to $E_{\text {coll }} \sim 37.0,39.5,46.1,48.6,56.0$, and $68.6 \mathrm{~cm}^{-1}$. The small shoulder at $E_{\text {coll }} \sim 26.5 \mathrm{~cm}^{-1}$, i.e., below the threshold of the $0_{00} \rightarrow 2_{02}$ transition, is also well reproduced. As a result, all main experimental features can be attributed to a theoretical counterpart. The origin of the six main resonances is now to be investigated.

The contributions from each partial wave to the integral cross section $\sigma\left(E_{\text {coll }} ; 0_{00} \rightarrow 2_{02}\right)$ are plotted in Fig. 3 as function of collisional energy. These partial cross sections differ by their total angular momentum $J$. It is observed that for each resonance, one or at most two partial waves contribute significantly, which is characteristic of shape resonances, since they occur when a metastable state is trapped inside a specific centrifugal barrier. Indeed, many total angular momenta $J$ should contribute to Feshbach type resonances [24], as they indicate interferences between several output channels. In addition, the widths of the six peaks increase with energy, which is also typical of shape resonances. We have also checked the shape character by performing a three-state calculation (including only $0_{00}, 1_{11}$ and $2_{02}$ ), in which coupling to excited closed channels is missing. The resonance structure with six peaks was found 


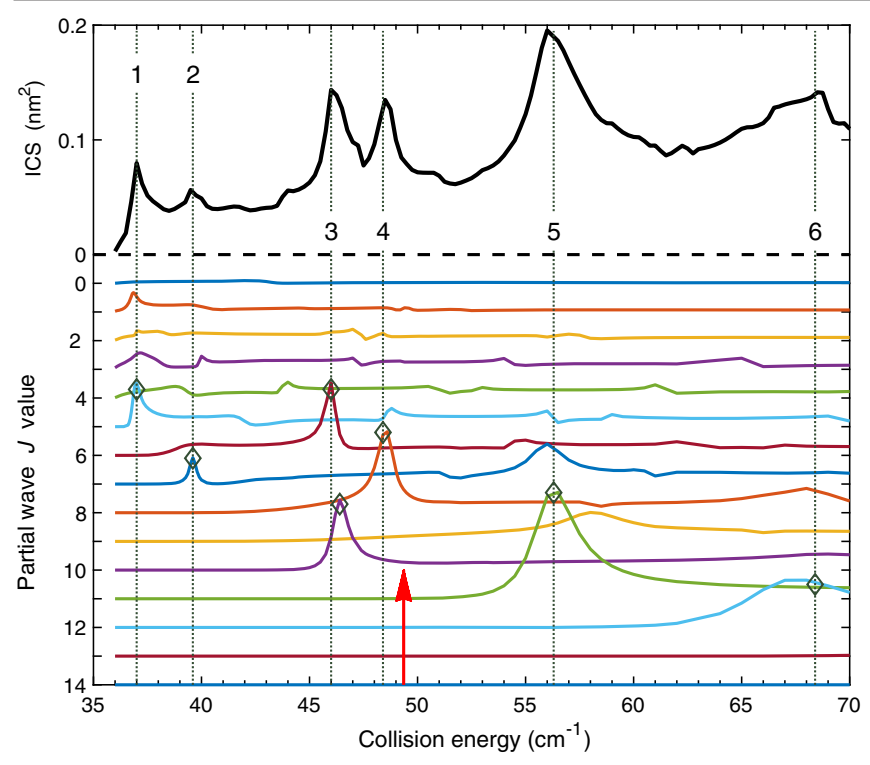

FIG. 3. Partial cross sections $\sigma^{J}\left(E_{\text {coll }}\right)$ for the $0_{00} \rightarrow 2_{02}$ transition of ortho- $\mathrm{D}_{2} \mathrm{O}$ in collisions with para- $\mathrm{H}_{2}\left(j_{2}=0\right)$. Partial wave $J$ values are given on the left vertical axis. Note that all partial cross section scales are identical, but that of the integral cross section is different. Positions of the resonances are indicated by vertical dashed lines and diamonds give the main contributions, analyzed in the Supplemental Material, Fig. 2. The red arrow indicates the opening of the $0_{00} \rightarrow 2_{11}$ channel.

to remain, although it was shifted to lower energies and the background cross section was reduced by a factor of $\sim 2$ (because of the reduced rotational basis of $\mathrm{H}_{2}$, keeping only $j_{2}=0$ ). It is remarkable to note that in these partial cross sections, the opening of a new channel (red arrow in Fig. 3) seems to have no importance at all. It shows that both the threshold energy and the centrifugal energy contribute to the position of the resonance, seen here as a metastable state of the effective potential, i.e., the PES plus the centrifugal term. Finally, the convergence is achieved at $J=12$, with $J>12$ contributions being without features and decreasing exponentially with $J$.

We have also computed the DCSs in the vicinity of the six resonance peaks, at resonance and off-resonance energies. These are shown in Fig. 2 of Supplemental Material. The behavior of the DCSs is not clear-cut, with no evident preference for forward or backward scattering. We observe, however, an obvious increase of both forward and backward scattering at the resonance energy. Backward scattering dominates at peaks 3,5 while forward scattering dominates at peaks $1,2,4,6$. In the simpler $\mathrm{NH}_{3}-\mathrm{He}$ system, increased backward scattering was observed at energies close to a resonance [25]. The structure of the DCS near a resonance is more difficult to predict in molecule- $\mathrm{H}_{2}$ systems, as discussed by Ma et al. [24] in the case of $\mathrm{NH}_{3}-\mathrm{H}_{2}$. One can also notice that the behavior of each of the main partial waves is increasingly oscillating as the total angular quantum number $J$ is increasing, indicating more interfering terms in the DCS, consistent with the impact of $J$ on the behavior of the DCS. We are thus assigning a shape nature to all the six resonances analyzed here.

Conclusion.-With a crossed molecular beam setup dedicated to low energy collisions, we succeeded in measuring the structure of the rotationally inelastic collisions between $\mathrm{D}_{2} \mathrm{O}$ and $\mathrm{H}_{2}$, at collisional energies between 15 and $105 \mathrm{~cm}^{-1}$. The selectivity of the initial conditions (rotational ground states of both $\mathrm{D}_{2} \mathrm{O}$ and $\mathrm{H}_{2}$ ), energy resolution, and the single collision conditions allowed us to detect in four bumps, the six main resonance peaks predicted theoretically. These peaks, calculated with a fully converged quantum computation based on a fulldimensional water-molecular hydrogen PES adapted here to the $\mathrm{D}_{2} \mathrm{O}-\mathrm{H}_{2}$ isotopic variant, were faithfully reproduced, both in absolute positions and relative intensities. An analysis in terms of partial waves points to resonances due to metastable states in the effective potential energy compounded by the PES and the centrifugal energy, so-called shape resonances. This joined experiment and theory investigation shows the great quality of the V08 PES and the subsequent converged dynamics. It allows one to have full confidence in observables computed with this PES, including rotational (de)excitation rate coefficients for astrophysics, transport properties for water diluted in molecular hydrogen, or the dynamics of molecular hydrogen confined in the cages of clathrate hydrates [26].

Nicole Feautrier is acknowledged for angular algebra insights. This research was partly supported by the Programme National "Physique et Chimie du Milieu Interstellaire" (PCMI) of CNRS (INSU, INC, and INP departments), co-funded by CEA and CNES. Part of this work was supported by the Institut Pascal, Université ParisSaclay, DYMCOM project. Computations were performed using the CNRS-IDRIS national computers, Program No. A0060810769 or using the GRICAD infrastructure [28], which is supported by Grenoble Research Communities.

*astrid.bergeat@u-bordeaux.fr

[1] A. Baudry, E. M. L. Humphreys, F. Herpin, K. Torstensson, W. H. T. Vlemmings, A. M. S. Richards, M. D. Gray, C. De Breuck, and M. Olberg, Astron. Astrophys. 609, A25 (2018).

[2] E. F. van Dishoeck, E. Herbst, and D. A. Neufeld, Chem. Rev. 113, 9043 (2013).

[3] H. M. Butner, S. B. Charnley, C. Ceccarelli, S. D. Rodgers, J. R. Pardo, B. Parise, J. Cernicharo, and G. R. Davis, Astrophys. J. Lett. 659, L137 (2007).

[4] A. Faure, N. Crimier, C. Ceccarelli, P. Valiron, L. Wiesenfeld, and M. L. Dubernet, Astron. Astrophys. 472, 1029 (2007).

[5] F. Daniel, M.-L. Dubernet, and A. Grosjean, Astron. Astrophys. 536, A76 (2011).

[6] A. Faure, L. Wiesenfeld, Y. Scribano, and C. Ceccarelli, Mon. Not. R. Astron. Soc. 420, 699 (2012). 
[7] P. Valiron, M. Wernli, A. Faure, L. Wiesenfeld, C. Rist, S. Kedžuch, and J. Noga, J. Chem. Phys. 129, 134306 (2008).

[8] P. M. Felker, D. Lauvergnat, Y. Scribano, D. M. Benoit, and Z. Bačić, J. Chem. Phys. 151, 124311 (2019).

[9] L. Belpassi, M. L. Reca, F. Tarantelli, L. F. Roncaratti, F. Pirani, D. Cappelletti, A. Faure, and Y. Scribano, J. Am. Chem. Soc. 132, 13046 (2010).

[10] Y. Scribano, O. Akin-Ojo, and A. Faure, J. Chem. Phys. 135, 116101 (2011).

[11] M. P. Ziemkiewicz, C. Pluetzer, D. J. Nesbitt, Y. Scribano, A. Faure, and A. van der Avoird, J. Chem. Phys. 137, 084301 (2012).

[12] B. Drouin and L. Wiesenfeld, Phys. Rev. A 86, 022705 (2012).

[13] C. Yang, G. Sarma, J. J. Ter Meulen, D. H. Parker, G. C. McBane, L. Wiesenfeld, A. Faure, Y. Scribano, and N. Feautrier, J. Chem. Phys. 133, 131103 (2010).

[14] C.-H. Yang, G. Sarma, D. H. Parker, J. J. ter Meulen, and L. Wiesenfeld, J. Chem. Phys. 134, 204308 (2011).

[15] G. Sarma, C.-H. Yang, A. K. Saha, D. H. Parker, and L. Wiesenfeld, J. Chem. Phys. 138, 024314 (2013).

[16] A. Bergeat, A. Faure, S. B. Morales, A. Moudens, and C. Naulin, J. Phys. Chem. A 124, 259 (2020).

[17] A. Bergeat, S. B. Morales, C. Naulin, J. Klos, and F. Lique, F. Lique., Front. Chem. 7, 164 (2019).

[18] C. M. Western and B. E. Billinghurst, Phys. Chem. Chem. Phys. 21, 13986 (2019).
[19] C. H. Yang, G. Sarma, J. J. Ter Meulen, D. H. Parker, and C. Western, Phys. Chem. Chem. Phys. 12, 13983 (2010).

[20] J. M. Hutson and S. Green, Molscat Computer Code, Version 14, distributed by Collaborative Computational Project 6 (Daresbury Laboratory, Warington, UK, 1995).

[21] Y. Scribano, A. Faure, and L. Wiesenfeld, J. Chem. Phys. 133, 231105 (2010).

[22] We found an error in the implementation of Ref. [21] so that in practice, the V08 PES routine was used in this work to generate a new rigid-rotor, five-dimensional, $\mathrm{D}_{2} \mathrm{O}-\mathrm{H}_{2}$ PES.

[23] T. Stoecklin, O. Denis-Alpizar, A. r. Clergerie, P. Halvick, A. Faure, and Y. Scribano, J. Phys. Chem. A 123, 5704 (2019).

[24] Q. Ma, A. van der Avoird, J. Loreau, M. H. Alexander, S. Y. T. van de Meerakker, and P. J. Dagdigian, J. Chem. Phys. 143, 044312 (2015).

[25] K. B. Gubbels, S. Y. T. van de Meerakker, G. C. Groenenboom, G. Meijer, and A. van der Avoird, J. Chem. Phys. 136, 074301 (2012).

[26] See Supplemental Material at http://link.aps.org/ supplemental/10.1103/PhysRevLett.125.143402 for some theoretical and experimental detailed descriptions, which includes Ref. [27].

[27] S. T. Pratt, P. M. Dehmer, and J. L. Dehmer, Chem. Phys. Lett. 105, 28 (1984).

[28] https://gricad.univ-grenoble-alpes.fr 